\title{
Assessment of marginal degradation of restorations on impressions
}

\author{
Asbjörn Jokstad and Ivar A. Mjör \\ Department of Anatomy, School of Dentistry, University of Oslo, Blindern, Oslo, and \\ NIOM, Scandinavian Institute of Dental Materials, Haslum, Norway
}

\begin{abstract}
Jokstad A, Mjör IA. Assessment of marginal degradation of restorations on impressions. Acta Odontol Scand 1991;49:15-25. Oslo. ISSN 0001-6357.

The study aimed to validate the scoring of the degree of marginal degradation of amalgam restorations by using impressions, as an alternative to other indirect scoring methods using photographs or casts. Ten-year-old condensation silicone elastomer impressions and epoxy replicas made in 1979 were compared in a scanning electron microscope at $5 \mathrm{kV}$ with different magnifications up to $\times 200$. The impression material was not distorted or degraded, and the dimensional stability was good after 10 years of storage in a dry environment. The interexaminer agreement of the scorings of impressions and a six-point scale reference set was satisfactory as evaluated by kappa statistics, demonstrating that degrees of marginal degradation can be distinguished on impressions with relatively high accuracy. The rating distribution of the scorings of impressions showed good correlation to the rating distributions obtained with the clinical USPHS rating method and with photographs for recording marginal degradation. A slight difference between the photographic and impression ratings at the upper and lower levels of the six-point rating scale was observed. The difference varied with the type of alloy, possibly due to a bias depending on the surface quality-that is, whether the restoration kept the glossiness of high polishing or became heavily tarnished. $\square$ Amalgam degradation; clinical rating; clinical study; kappa statistics; silicone elastomer
\end{abstract}

Asbjörn Jokstad, Department of Anatomy, Dental Faculty, P.O. Box 1052 Blindern, University of Oslo, $\mathrm{N}$-0316 Oslo 3, Norway

Various indirect clinical techniques for rating or ranking the marginal adaptation of restorations has been described in the literature. The commonest technique is the scoring using intraoral black and white photographs at $\times 7$ magnification $(1,2)$. Many investigators have also used color photographs or color slides varying from $\times 1.5$ up to $\times 52$ (3). Other investigators have used impressions from which replicas are made. The replicas have been observed (4), photographed (5-7), assessed in a profile recorder $(8,9)$ or a scanning electron microscope (SEM) (10), or measured quantitatively by other methods $(10-13)$. The use of photographs or models for the assessments of clinical marginal adaptation has been validated in several studies $(14,15)$, but there are advantages and disadvantages involved in using the different recording techniques (Tables 1 and 2).

There is a lack of information in the literature on the relationship between findings of controlled clinical studies and the clinical performance in field trials $(31,32)$. In field trials the recording is made by a non-specialized staff in their normal clinical practice. The trial protocol must therefore exclude technique-sensitive recording methods and high-caliber equipment (33). In addition, the clinical recording procedures should be fast and simple, to obtain continuous cooperation with clinicians and patients. Although scoring of the marginal degradation on photographs is relatively simple for the evaluators, the photographic recording is not optimal in field trials, since it is time-consuming and requires training of the clinical staff (Table 1).

The alternative indirect recording technique of assessing the marginal degradation by using replicas seems beneficial, since minimal training of personnel is required in the procedures for taking impressions. A disadvantage of the method is the step of making casts of the impressions, which 
Table 1. Photographic technique

\begin{tabular}{|c|c|}
\hline Advantages & Disadvantages \\
\hline $\begin{array}{l}\text { A magnification considerably greater than the } \\
\text { normal tooth size contributes significantly to a } \\
\text { more accurate interpretation (16) } \\
\text { Produces a permanent record of the restoration (17) } \\
\text { The raw data are always available for } \\
\text { reexamination, change in evaluators or } \\
\text { modification in evaluation methods (17) }\end{array}$ & $\begin{array}{l}\text { Difficulty in obtaining consistently acceptable } \\
\text { pictures (1) } \\
\text { Takes time to photograph each situation so all } \\
\text { occlusal margins of the restoration are } \\
\text { observed (1) } \\
\text { Requires high-caliber equipment and the use of } \\
\text { mirrors so the long axis of the tooth and the } \\
\text { camera lens are parallel (1) } \\
\text { The restoration must be thoroughly dried and } \\
\text { plaque-free (1) } \\
\text { Important to have a standardized distance, } \\
\text { direction, and illumination for reproducible } \\
\text { accuracy (18, 19) } \\
\text { Not necessarily correlated to clinical ratings (20) } \\
\text { The three-dimensional nature of the marginal } \\
\text { crevice cannot be evaluated with a two- } \\
\text { dimensional photographic method, since the } \\
\text { width of the ditch correlates with the depth } \\
\text { less than } 35 \% \text { measured by } \\
\text { stereophotogrammetry (20, } 21) \\
\text { Double-blind studies may be impossible since } \\
\text { examiners distinguish the different materials } \\
\text { visually. Trained clinicians will recognize the } \\
\text { alloys due to the surface tarnish (22) } \\
\text { Proximal marginal deficiencies poorly } \\
\text { represented (23) } \\
\text { Difficult to demonstrate clearly the extent of } \\
\text { material loss of a composite material due to } \\
\text { ability to absorb and reflect color or shading } \\
\text { of the surrounding structure (16) }\end{array}$ \\
\hline
\end{tabular}

increases the preparation time and may possibly also introduce artefacts (34). The necessity of including an extra processing step also contradicts the basic aim of all study protocols-that is, to keep the number of necessary work procedures at a minimum to provide accurate accounts of the gathered data (16).

Little consideration has been given to the possibility of evaluating the marginal degradation directly on the impressions (35). The method of scoring marginal degradation directly on the impressions has only been described in one report (7). This method requires that the marginal discrepancies can be recognized with ease. The ratings should also show a fair comparison with other validated indirect clinical assessment techniques or with a direct clinical assessment technique. Furthermore, permanent records of the restorations require dimensional stability of the impression material over years of storing. In addition, the impression material should be sufficiently rigid to withstand the preparation for, and examination in, a scanning electron microscope without loss of details.

The object of the study was to examine the correlation between the scorings of the marginal degradation by means of impressions made of silicon elastomers and the scorings obtained by means of a clinical technique and by means of photographs. Furthermore, we wanted to establish the sensitivity of the scoring technique by determining the inter-examiner agreement for three examiners. A further aim of the study was to observe the impressions in a scanning electron microscope and compare the surface topography and the dimensional stab- 
Table 2. Model or replica technique

\begin{tabular}{|c|c|}
\hline Advantages & Disadvantages \\
\hline $\begin{array}{l}\text { Permanent record of the restoration (17) } \\
\text { The raw data are always available for } \\
\text { reexamination, change in evaluators } \\
\text { or change in evaluation methods (17) } \\
\text { Very fast technique of registration if the } \\
\text { impression material is loaded on the } \\
\text { end of a tongue spatula and inverted } \\
\text { over the tooth. Thus also applicable } \\
\text { on children }(22,24) \\
\text { Defects are revealed more prominently } \\
\text { on casts than on the photographs } \\
\text { owing to the possibility of rotating the } \\
\text { specimen (1) } \\
\text { Allows cross-checking with clinical } \\
\text { findings (25) } \\
\text { Allows possible SEM investigations } \\
\text { when appropriate (25) }\end{array}$ & $\begin{array}{l}\text { A suitable material to fill the negative } \\
\text { impression has up to recently been lacking. } \\
\text { Air bubbles or chemical interaction between } \\
\text { impression material and replica material } \\
\text { frequent occurrence (26-28) } \\
\text { Linear dimensional changes during setting may } \\
\text { vary with different epoxy resins (29) } \\
\text { Not necessarily correlated to clinical ratings (20) } \\
\text { The translucency and color of epoxy materials } \\
\text { make observations difficult (22) } \\
\text { Impression material is torn if the coronal part is } \\
\text { retained in the margin. The casts may thus } \\
\text { have artificially shallow marginal crevices ( } 30) \\
\text { Potential marginal deficiencies proximally are } \\
\text { poorly represented (23) }\end{array}$ \\
\hline
\end{tabular}

ility of 10-year-old impressions and of 10year-old replicas made of an epoxy material from the same impressions.

\section{Materials and methods}

\section{SEM}

The impressions and the epoxy casts of class-II preparations were made in 1979 and had since then been stored in microscope slide boxes in a dry environment. The impression materials were condensation silicon elastomers (Xantropren blue and Optosil, Bayer, Leverkusen, FRG). These materials had been selected because of their high resolution $(26,35,36)$. Casts of the impressions had been made within $72 \mathrm{~h}$, using an epoxy material (Durcupan, Fluka AG, Buchs, Switzerland). The impressions and the epoxy casts were made conductive by coating with $10-\mathrm{Nm}$ platinum in a diode sputter coater with a cooled target and specimen stage (Polaron type E 5100, Polaron Equipment Ltd., Watford, Herts, England). The impressions and epoxy casts were examined in a scanning electron microscope (SEM 515, Philips, Eindhoven, The Netherlands) between 2 and $20 \mathrm{kV}$ and at magnifications up to $\times 200$. The criterion for selecting specific impressions and epoxy casts for observations in the SEM was an occlusal surface with easily recognizable anatomic details. The surface topography of the impression and of the epoxy model was assessed by comparing the same areas on the negative and positive replica. The dimensional stability of the impression material was estimated by measuring the distance between two identified surface details on the SEM micrographs of the impression and of the epoxy cast. The dimensions were also assessed before the coating procedures for the SEM in a stereomicroscope at $\times 20$ with a measuring ocular (Spencer American Optical).

\section{Clinical evaluation}

The material consisted of 468 2- and 3surface class-II restorations made from 5 different alloys at base line. The alloys used were Revalloy (SS White Ltd., U.K.), Indiloy (Shofu Dental Corp., Japan), Tytin (SS White Ltd., U.K.), Dispersalloy (Johnson \& Johnson, USA), and Amalcap Non gamma 2 (Vivadent, FRG).

One hundred and ninety-two restorations were observed during 5 years. An impression and a black-and-white photograph were taken at base line after polishing, at 6 months, and each year up to 5 years. The clinicians $(n=3)$ were supplied with a copy of the first photograph to help in the stan- 


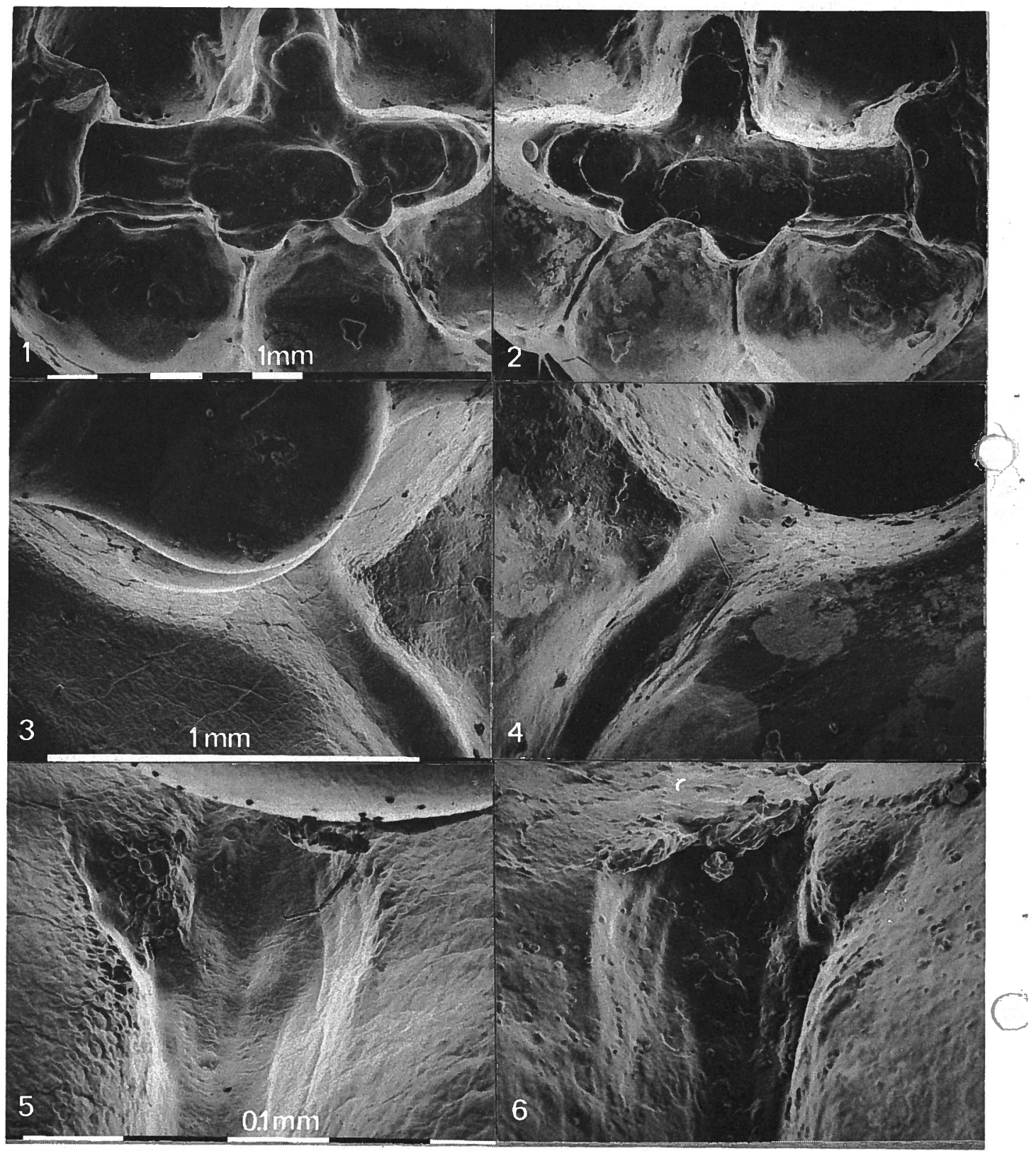

Figs. 1-6. SEM micrographs of a class-II cavity preparation made in 1979. Figs. 1, 3, and 5 are 10-year-old negative replicas made of a condensation silicone elastomer, while Figs. 2, 5, and 6 are 10-year-old positive replicas made of epoxy. Magnification: Figs. 1 and 2, $\times 10$; Figs. 3 and 4, distobuccal fissure on Fig. 1, $\times 75$; Figs. 5 and 6, mesiobuccal fissure on Fig. 1, $\times 200$. The light gray zones on the surface on the epoxy replicas are presumably caused by a chemical interaction between the impression material and the epoxy material at the time of casting. 
dardization of later photographs. The photographs were produced with a $200-\mathrm{mm}$ Medical Nikon lens (Nikon Inc., USA) at a magnification of $\times 1.5$. Prints were made to reproduce the original size approximately six times and cropped to show only the restored tooth. The materials used for impressions were identical to those described in the previous section. The impressions of the involved teeth were cut, mounted on a glass slide, and examined in a stereomicroscope at $\times 20$ (Spencer American Optical).

The marginal degradation observed on the photographs was scored in accordance with a reference set of photographs. The marginal degradation observed on the impressions was scored relative to a reference set of impressions. Both reference sets consisted of six categories with increasing extent of marginal degradation and equal intervals of perceptible difference in the extent of fracture. All scorings were made by a trained technician and a dentist. Any differences in the scorings between the two evaluators were solved by joint agreement on one value. The inter-examiner agreement on scoring impressions was assessed by three uncalibrated examiners evaluating a subsample of 50 impressions.

The scoring of the marginal degradation on the photographs was compared with the scorings in another sample, consisting of 277 restorations, using the same amalgams and photographed at the same observation intervals. These restorations had also been examined clinically and scored in accordance with the protocol of the USPH system (37). The USPHS scorings used were Alpha (crevice along the margin into which the explorer cannot penetrate), Beta (crevice into which the explorer will penetrate), and Charlie (margins with dentin or base exposed).

Kappa statistics was used to establish the inter-rating agreement of the scorings when using the impressions (38). A kappa index was also calculated for the scorings when using the photographs and when using the impressions across all categories and across each individual category of the full scale. In addition, the index was computed to detect any systematic biases in the evaluation of the individual amalgam alloys and to relate the indirect scoring methods to the USPHS ratings. All statistics were made on the cumulative scorings over 5 years.

\section{Results}

\section{SEM}

Occlusal fissures perpendicular to the restoration margins were well suited as reference details on the impression and on the equivalent epoxy cast. Measurements between two such details in the stereomicroscope at $\times 20$ showed that the epoxy models were slightly smaller than the impressions before these were coated. The proportional relationship was approximately 0.99 to 1 , and the precision of the measurements was \pm 0.01 . This proportional relationship was also measured directly on the SEM micrographs at the different magnification levels. SEM micrographs of one area are shown in Figs. $1-6$ at $\times 10, \times 75$, and $\times 200$ magnification levels. Thus, the procedure of coating the impressions did not seem to cause any significant shrinkage of the impression material.

The surface of the impression seemed smoother than the surface of the models, which became more apparent with increased magnification beyond $\times 75$. Owing to the lack of control material in the present study it is not possible to deduce whether this is a surface degradation of the elastomer, or whether the more irregular surfaces on the models are artefacts. Crazing of the conductive layer on the impression material could not be observed, even when electron beams up to $20 \mathrm{kV}$ were used. The prevailing artefacts on the impressions and epoxy replicas were pores and structureless areas. These were usually at a macroscopic level and were presumably the result of incomplete polymerization due to poor mixing of the materials. The typical artefacts at the microscopic level were dust particles. In addition, the epoxy replicas often showed multiple demarcated areas with different shadowing on the micrographs (Fig. 4). These areas were presumably caused by an interaction between the impression material and the epoxy resin at the time of casting. 


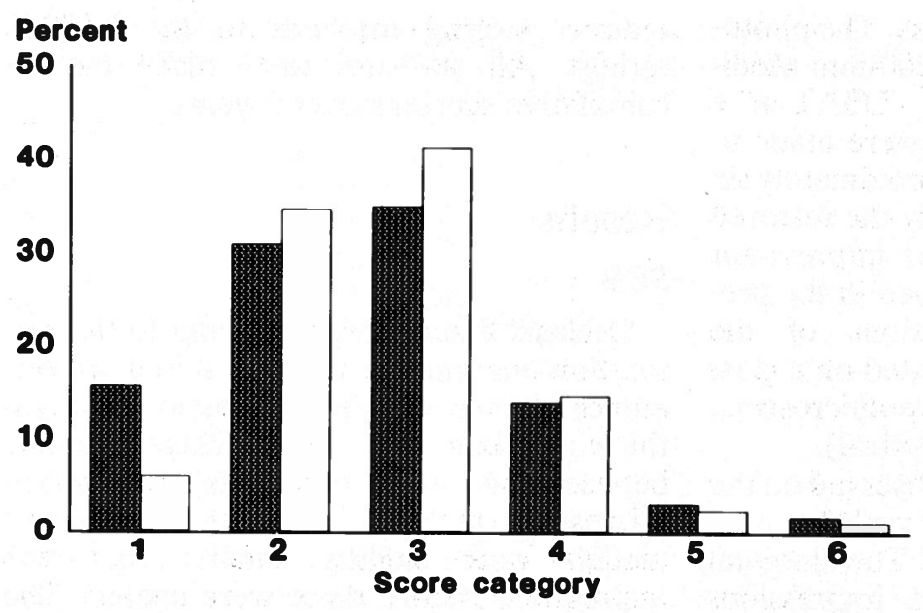

Fig. 7. The distribution of the ratings on marginal degradation of amalgam restorations $(n=192)$, using photographs (dark bar) and impressions (open bar). Rating 1 represents minimal extent of marginal degradation; rating 6 represents extent of degradation necessitating replacement of restoration.

There were no attempts to quantify the frequency of artefacts on the different types of replicas. The interpretation of the negative impression relative to the positive replica did not present a major difficulty in the orientation.

\section{Clinical evaluation}

The inter-examiner agreements between the three examiners were $k=0.41$ between examiner $A$ and examiner $B, K=0.49$ between examiner $B$ and examiner $C$, and $\kappa=0.47$ between examiner $A$ and examiner C. Examiners $\mathrm{A}$ and $\mathrm{C}$ were technicians trained to score marginal degradation on photographs, whereas examiner B was a dentist. The examiners were not calibrated before the impressions were scored, but the use of the scoring system was explained.

A comparison of the scorings of the marginal degradation when using the photographic and the impression techniques of the same 192 restorations is presented in Fig. 7. The central tendencies of the rating distributions are similar, but the photographic scorings are slightly more positively skewed. A cross-tabulation of the scorings obtained by the two techniques shows that the rating agreement varies slightly (Table 3 ). The scores were higher when the impressions were evaluated than when using the photographs at the low level of the rating scale; that is, the marginal degradation was scored as more severe when using the impressions. This also occurred at the high level of the

Table 3. Cumulative ratings of 192 restorations observed at base line, half year, and yearly up to 5 years by the use of photographs and by the use of impressions; $n=1194$

\begin{tabular}{|c|c|c|c|c|c|c|c|c|}
\hline \multirow{2}{*}{$\begin{array}{l}\text { Impression } \\
\text { rating }\end{array}$} & \multicolumn{6}{|c|}{ Photographic rating } & \multirow[b]{2}{*}{ Total } & \multirow[b]{2}{*}{ Proportion } \\
\hline & 1 & 2 & 3 & 4 & 5 & 6 & & \\
\hline 1 & 54 & 18 & & & & & 72 & 0.06 \\
\hline 2 & 128 & 230 & 52 & 6 & & & 416 & 0.35 \\
\hline 3 & 3 & 110 & 312 & 55 & 9 & & 489 & 0.41 \\
\hline 4 & & 9 & 55 & 92 & 16 & 4 & 176 & 0.15 \\
\hline 5 & & & 3 & 8 & 12 & 6 & 29 & 0.02 \\
\hline 6 & & & & & & 12 & 12 & 0.01 \\
\hline Total & 185 & 367 & 422 & 161 & 37 & 22 & & \\
\hline Proportion & 0.16 & 0.31 & 0.35 & 0.14 & 0.03 & 0.02 & & 1.00 \\
\hline
\end{tabular}


Table 4. Kappa scores of agreement for individual categories and across all categories of Table 3. Po $=$ proportion of observed agreement; $\mathrm{Pe}=$ proportion of chanceexpected agreement; $\mathrm{SE}=$ standard error

\begin{tabular}{lcccccc}
\hline & Po & Pe & Kappa & Kappa, SE & Z & $p$ \\
\hline Score 1 & 0.87 & 0.81 & 0.32 & 0.025 & 13 & $<0.0001$ \\
Score 2 & 0.73 & 0.57 & 0.37 & 0.030 & 12 & $<0.0001$ \\
Score 3 & 0.76 & 0.54 & 0.48 & 0.030 & 16 & $<0.0001$ \\
Score 4 & 0.87 & 0.75 & 0.48 & 0.029 & 17 & $<0.0001$ \\
Score 5 & 0.97 & 0.95 & 0.39 & 0.029 & 14 & $<0.0001$ \\
Score 6 & 0.99 & 0.97 & 0.66 & 0.027 & 24 & $<0.0001$ \\
Overall & 0.60 & 0.28 & 0.43 & 0.017 & 26 & $<0.0001$ \\
\hline
\end{tabular}

rating scale, while the relationship was inverse in the middle range (Table 3 ). The total agreement was kappa $=0.43$, and the kappa values varied from 0.32 to 0.66 on the rating scale (Table 4).

The total rating agreement of the two techniques varied with the type of alloy. High rating agreement was observed for Revalloy and Amalcap, while poor rating agreement was observed for Indiloy and Tytin (Table 5). The rating agreement also varied with the type of alloy at the individual category levels. The highest rating agreement was seen when the marginal degradation of Revalloy was scored (Table 5). The poor rating agreement at the low end of the rating scale was similar for all alloys. At the high end of the rating scale the differences were most marked for Revalloy and Dispersalloy (Fig. 8).

The indirect method of rating the restorations on photographs correlated well with the USPHS clinical ratings in the sample with the 277 restorations $(K=0.47)$. The proportions of the clinical scorings were compared with the six categorical rating groups. Good agreement could be observed for Alpha and the ratings 1,2 , and $3(k=$ $0.43)$, Bravo and the ratings 4 and $5(k=$ $0.43)$, and Charlie and rating $6(k=1)$. The rating distributions were similar when the clinical scorings and the impression technique were used in the group with the 277 restorations. The two rating distributions were also similar to the ratings measured by the two indirect techniques in the group with the 192 restorations (Fig. 9).

\section{Discussion}

Many impression materials have been used for replication of restorations surfaces, including polymethylmetacrylates (39),

Table 5. Kappa scores of agreement for individual categories and across all categories by the use of photographs and by the use of impressions for different alloys

\begin{tabular}{lccccc}
\hline & $\begin{array}{c}\text { Amalcap, } \\
n=303\end{array}$ & $\begin{array}{c}\text { Dispersalloy, } \\
n=266\end{array}$ & $\begin{array}{c}\text { Indiloy, } \\
n=120\end{array}$ & $\begin{array}{c}\text { Revalloy, } \\
n=386\end{array}$ & $\begin{array}{c}\text { Tytin, } \\
n=119\end{array}$ \\
\hline Score 1 & 0.39 & 0.31 & 0.14 & 0.50 & 0.38 \\
Score 2 & 0.54 & 0.26 & 0.13 & 0.40 & 0.28 \\
Score 3 & 0.49 & 0.42 & 0.48 & 0.51 & 0.49 \\
Score 4 & 0.38 & 0.56 & 0.22 & 0.48 & 0.66 \\
Score 5 & 0.38 & 0.31 & & 0.52 & \\
Score 6 & & 0.65 & & 0.49 & \\
Overall & 0.47 & 0.40 & 0.23 & 0.48 & 0.39 \\
SE & 0.036 & 0.035 & 0.055 & 0.028 & 0.064 \\
Z value & 13.3 & 11.4 & 4.1 & 17.2 & 6.0 \\
$p$ & $<0.0001$ & $<0.0001$ & $<0.0001$ & $<0.0001$ & $<0.0001$ \\
\hline
\end{tabular}




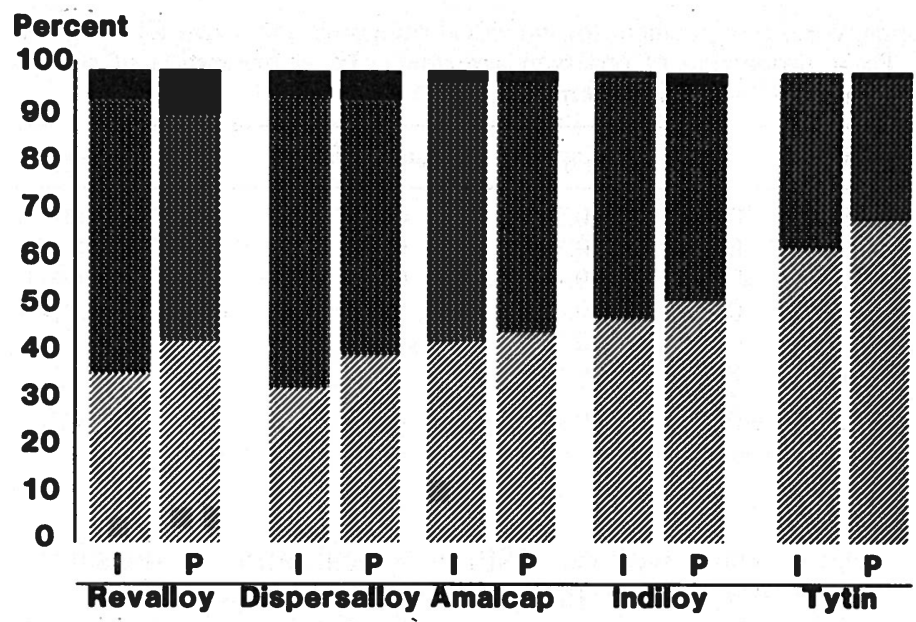

Fig. 8. The cumulative percentages of ratings on marginal degradation of amalgam restorations using impressions (I) and photographs (P) by different alloys. The alloys are Revalloy $(n=386)$, Dispersalloy $(n=266)$, Amalcap $(n=303)$, Indiloy $(n=120)$, and Tytin $(n=119)$. Rating 1 represents minimal extent of marginal degradation; rating 6 represents extent of degradation necessitating replacement of

restoration. Ratings 1 and 2 are represented by the light shadow, ratings 3 and 4 by the dark shadow, and ratings 5 and 6 by black.

celluloid (40), and several common dental impression materials $(27,34)$. A condensation silicone elastomer was chosen because of its high resistance and good detail reproduction (41). New epoxy materials made from the condensation silicone elastomer Xantopren blue have been shown to resolve features down to $0.3 \mu \mathrm{m}$ in $\operatorname{SEM}(28,42)$. A drawback of the material is that Xantopren blue and other condensation silicone elastomers contract $0.1 \%$ to $1 \%$ over $72 \mathrm{~h}$ during the polymerization (43). Investigators studying the high resolution or the topography of surfaces therefore recommend that casts $(44$, $45)$, electroplating $(46,47)$ or laser measurements (48) should be made shortly after taking the impressions. However, the sizes of marginal ditches are usually in the order

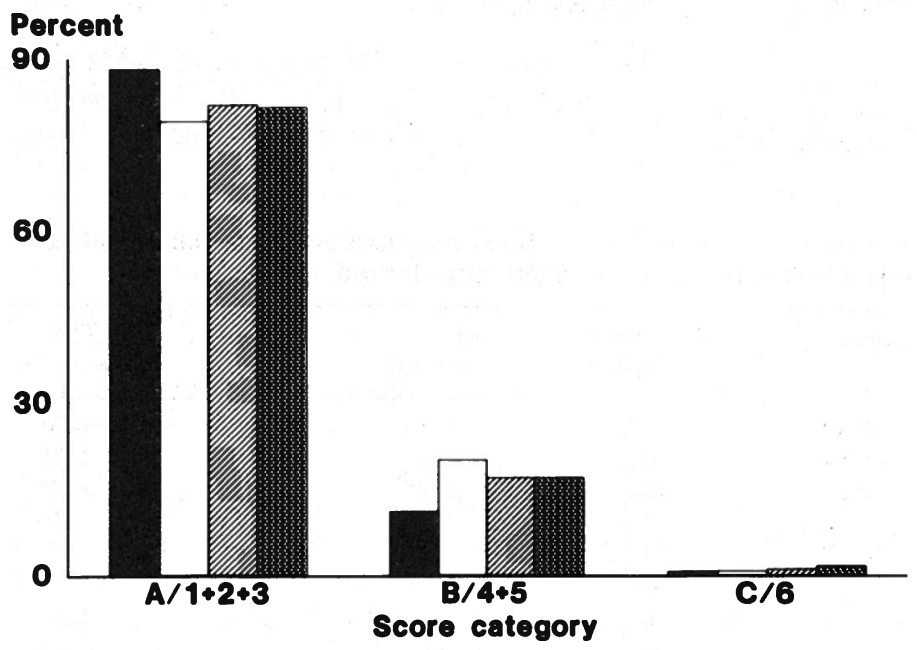

Fig. 9. The rating distribution of the marginal degradation of 277 amalgam restorations using the USPHS clinical method (closed bars) and photographs (open bars), compared with the rating distribution of 192 restorations assessed by using impressions (bars with light shadow) and photographs (bars with dark shadow). The data are pooled as USPHS scorings Alpha $=$ crevice along the margin into which the explorer cannot penetrate; $\mathbf{A}=$ ratings $1+2+3 ;$ Beta $=$ crevice that the explorer will penetrate; $B=4+5 ;$ and Charlie $=$ margins with dentin or base exposed; $C=6$. 
of 10 to $400 \mu \mathrm{m}(23,49)$. It is therefore questionable whether a 1-2\% contraction of the impression material could influence the scoring of marginal degradation, as long as the impression is not grossly distorted.

The impressions were slightly larger than the epoxy casts, and the relative dimensional proportion was similar in the stereomicroscope and in the SEM. The epoxy material used in the present study has been shown to contract 6\% during setting (29). If the epoxy material is stable in the SEM, it is probable that the impressions reproduce the tooth dimensions more correctly than the casts in the present study. The results thus show that the impression material does not distort and is adequately stable over 10 years. The timeand cost-consuming procedure of replicating the silicone elastomer impressions with epoxy plastic can therefore be avoided. One reason the impressions are frequently replicated with epoxy materials is that the silicon elastomers were believed to be unsuitable for SEM studies (36). Previous studies showed a crazing of the conductive layer on silicone impressions because of deformation in the vacuum $(27,50)$. Other studies have shown that when the specimens are coated with a thicker layer of metal than usual, they can be viewed at low magnification or with less than $10 \mathrm{kV}$ in the SEM (36). The thermal influence during the sputter coating procedure has been shown to affect the surface of the specimen (42). However, the present results show that when a diode sputtercoater with a cooled target and specimen stage is used, and platinum is used as a coating medium, satisfactory specimens are produced. Although magnifications as low as $\times 200$ at $5 \mathrm{kV}$ were used in the present study, higher magnification should be obtainable with modern scanning electron microscopes operating with electron currents down to $0.3 \mathrm{kV}$.

Frequently used indices for inter-examiner agreement are the percentage agreement and Pearson's correlation coefficient. These indices may be misleading, and kappa statistics were therefore chosen (51). The kappa statistic is a measure of the proportion of agreement beyond chance which is actually achieved. Kappa values between 0.40 and
0.75 represent fair to good agreement beyond chance. Values less than 0.40 represent poor agreement beyond chance (52). The inter-examiner agreements in the present study were not especially high. However, when considering that the examiners were not calibrated at the time of the scoring, the agreement rates are satisfactory. The results thus show that examiners may distinguish degrees of marginal degradation on impressions with relatively good accuracy. Other investigators have also observed that the discrimination potential of occlusal wear is identical on impressions and stone or epoxy resin replicas (53).

Statistical inferences of the scorings of marginal degradation have been obtained by using categorical rating scales and statistical methods for rates and proportions or by ranking the data with subsequent nonparametric statistical procedures $(1,54)$. Although a categorical scale was used in the present study, the principle of ranking impressions should be identical with ranking photographs. The disadvantage of using rating scales is that considerable variation in ratings for the same restorations may be given by different assessors $(55,56)$. Some investigators therefore consider measuring the degree of conformity in classifying each restoration unnecessarily rigorous for validating a method or measuring the interexaminer agreement (57). Instead, the accuracy of the central tendency value similar to a previously validated classification system should be used $(7,57)$. If this criterion is used for validation, the method of scoring marginal degradation on impressions thus can be supported, since the scoring on the impressions compared relatively well with the photographic and the clinical scores (Figs. 7 and 9).

The advantage of using the kappa statistics is that besides measuring the association between the two rating methods, it is possible to detect how the scoring methods deviate for each rating category. Earlier investigations have demonstrated that the rating of marginal degradation by means of photographs usually results in higher values than when models are used $(7,58)$. This relationship was also observed between photo- 
graphic and impression techniques in the present study, although only at the mid-level scoring categories. When, on the other hand, the restorations are at an early stage with clinically negligible marginal degradation or the alloys exhibit little marginal degradation, the scoring on impressions produced higher values-that is, more breakdown was recorded-than when scoring on the photographs. The situation was similar, but to a lesser extent, in the high rating categories. Some difference may be explained by the lack of using the same restorations to represent the six categories in both reference sets. This effect was considered small, since both sets were based on equal intervals of perceptible difference in the extent of degradation. Although the differences between the two rating techniques were not statistically significant, the trend was similar for all alloys (Table 5, Fig. 8). An inter-examiner variation in the rating at different levels on the rating scale has also been described when photographs have been used (59). It is possible that the variation observed in that study (59) and the variation between the photographic and impression rating techniques observed in the present study are the result of a biased rating of restorations if the surfaces remain highly polished throughout the observation time or if the surfaces become heavily tarnished or discolored, conditions that are only noted on the photographs.

\section{References}

1. Osborne JW, Phillips RW, Gale EN, Binon PP. Three-year clinical comparison of three amalgam alloy types emphasizing an appraisal of the evaluation methods used. J Am Dent Assoc 1976;93: 784-9.

2. Mahler DB, Terkla LG, Eysden Van J. Marginal fracture of amalgam restorations. J Dent Res 1973;52:823-7.

3. Smales RJ. Evaluation of clinical methods for assessing restorations. J Prosthet Dent 1983;49:6770.

4. Santucci EA, Racz WB, Norman RD. A new method for evaluating posterior composite restorations. IADR program and abstracts, 1979: Abstract no. 348.

5. Mitchem JC. Correlation of laboratory testing to three-year clinical behavior of silicate cements. J Prosthet Dent 1972;27:172-5.
6. Richter WA, Mahler DB. Physical properties vs clinical performance of pure gold restorations. J Prosthet Dent 1973;29:434.

7. Mjör IA, Ryge G. Comparison of techniques for the evaluation of marginal adaptation of amalgam restorations. Int Dent J 1981;31:1-5.

8. Smales RJ, Creaven PJ. Evaluation of clinical methods for assessing the surface roughness of restorations. J Prosthet Dent 1979;42:45-52.

9. Mahler DB, Van Eysden J. Occlusal extrusion of clinical amalgam restorations. IADR program and abstracts 1974:Abstract no. 26.

10. Lutz F, Imfeld T, Meier C, Firestone AR. Composite vs amalgam. Comparative measurements of in vivo wear resistance. 1 year report. Quintessence Int 1979;10:77-87.

11. Eick JD, Ortman LF, Allen TB, McGivern RF. General clinical application of stereo-camera system. IADR program and abstracts 1973:Abstract no. 642 .

12. Elderton RJ. An objective method for measuring the surface morphology of cavities and restorations in vivo. J Oral Rehabil 1977;4:323-33.

13. Miller BH, Marker VA, Spears R, Okabe T. Comparison of methods to evaluate breakdown in amalgam. IADR program and abstracts 1988:Abstract no. 1550.

14. FDI, Federation Dentaire Internationale. Recommendations for clinical research protocols for dental materials. Int Dent J 1982;32:403-23.

15. Baume LJ, Prejean F, Holz J. Les possibilites d'une normalisation des essais cliniques concernant les produits d'obturation dentaire. SSO 1976;86:60729.

16. Leinfelder KF. Methodological aspects of clinical research. In: Transactions of the international symposium on operative dentistry. Nijmegen: University of Nijmegen, 1975:87-103.

17. Jacobsen PH. The clinical evaluation of amalgam alloys. Br Dent J 1984;157:436-39.

18. Matsuda N. Marginal fracture of amalgam restorations. J Prosthet Dent 1970;23:658-61.

19. Herr P, Ciucchi B, Holz J, Baume LJ. Methode de positionnement de repliques destinee au controle clinique des materiaux d'obturation. J Biol Buccale 1981;9:17-26.

20. Smales RJ, Creaven PJ. Evaluation of three clinical methods for assessing amalgam and resin restorations. J Prosthet Dent 1985;54:340-6.

21. Eick JD, Ryge G, Tonn EM, Jendresen MD. Comparison of clinical evaluation method for amalgam IADR program and abstracts 1983:Abstract no. 63 .

22. Bates JF, Douglas WH. A two-year field trial of a disperse phase alloy. Br Dent J 1980;149:133-6.

23. Roulet JF, Reich T, Blunck U, Noack M. Quantitative margin analysis in the scanning electron microscope. Scanning Microsc 1989;3:147-59.

24. Davies NE, Tranter T, Whitten JR. Evaluation of fissure sealant durability in vivo using an impression technique. J Dent 1975;3:153-6.

25. Knibbs PJ, Plant CG, Shovelton DS, Jones PA. an evaluation of a lathe-cut high-copper amalgam alloy. J Oral Rehabil 1987;14:465-73. 
26. Pameijer $\mathrm{CH}$. Replica techniques for scanning electron microscopy-a review. Scan Electron Microsc $1978 ; 2 ; 831-4$.

27. Barnes IE. Replication techniques for the scanning electron microscope. 1. History, materials and techniques. J Dent 1978;6:327-41.

28. Scott EC. Replica production for SEM. A test of materials suitable for use in field settings. J Microsc 1982;125:337-341.

29. Ekfeldt A, Flöystrand F, Öilo G. Replica techniques for in vivo studies of tooth surfaces and prosthetic materials. Scand J Dent Res 1985;93:560-5.

30. Barnes IE. Replication techniques for the scanning electron microscope. 2. Clinical and laboratory procedures: interpretation. J Dent 1979:1;25-37.

31. NIDR, National institute of dental research. Challenges for the eighties National institute of dental research long range research plan. 1983;FY 198589.

32. Greener EH. Amalgam: Reactor response. Adv Dent Res 1988;2:83-86.

33. Møller IJ. The implementation of field trials. J Dent Res 1977;56C:C136-41.

34. Pameijer CH. Replica techniques. In: Hayat MA, ed. Principles and techniques of scanning electron microscopy. Biological applications. Vol. 4. New York: Van Nostrand Reinhold Co., 1974:45-92.

35. Kusy RP, Leinfelder C. In situ replication techniques. 1 . Preliminary screening and the negative replication technique. J Dent Res 1977;56:925-32.

36. Pameijer CH. Replication techniques with new dental impression materials in combination with different negative impression materials. Scan Electron Microsc 1979;2:571-4.

37. Cvar JF, Ryge G. Criteria for the clinical evaluation of dental restorative materials. Washington DC: U.S. Government Printing Office 1971;790 244:39.

38. Fleiss JL. The measurement of interrater agreement. In: Fleiss JL, ed. Statistical methods for rates and proportions. 2nd ed. New York: John Wiley \& Sons, 1981:211-36.

39. Roulet JF. Die Prüfung des Randschlusses sogenannt schnitzbarer Komposits. SSO 1978;88:34564.

40. Tomita Y, Okuda R, Wakumoto S. Replica technique for scanning electron microscope examination of occlusal amalgam margins. J Prosthet Dent 1979;41:299-303.

41. Phillips RW. Skinner's science of dental materials. 7th ed. Philadelphia: W.B. Saunders Co., 1978.

42. Vossen M, Letzel H, Stadhouders AM, Hertel R, Hendriks FH. A rapid SEM replication technique for clinical studies of dental restorations. Dent Mater 1985;1:158-63.
43. Marcinak CF, Draughn RA. Linear dimensional changes in addition curing silicone impression materials. J Prosthet Dent 1982;47:411.

44. Marker VA, Staman JW, Okabe T, Frederick K. Replication technique for high magnification resolution. IADR program and abstracts 1984:Abstract no. 1105 .

45. Marker VA, Nelson P, Filler WH, Okabe T. Variables affecting the quality of replicas for clinical investigations. IADR program and abstracts 1987: Abstract no. 834.

46. Lambrechts $P$, Vanherle $C$. A universal and accurate replica technique for SEM study in clinical dentistry. Microsc Acta 1981;85:45.

47. Lambrechts $P$, Vanherle G, Vuylsteke M, Davidson CL. Quantitative evaluation of the wear resistance of posterior dental restorations: a new three dimensional measuring technique. J Dent 1984;12:25267.

48. Atkinson JT, Groves D, Lalor MJ, Cunningham J, Williams DF. The measurement of wear in dental restorations using laser dual-source contouring. Wear 1982;76:91-104.

49. Fukushima M, Setcos JC, Phillips RW. Marginal fracture of posterior composite resins. J Am Dent Assoc 1988;117:577-583.

50. Pfefferkorn G, Boyde A. Review of replica techniques for scanning electron microscopy. Scan Electron Microsc 1974;1:75-82.

51. Hunt RJ. Percent agreement, Pearson's correlation, and Kappa as measures of inter-examiner reliability. J Dent Res 1986;65:128-30.

52. Landis JR, Koch GG. The measurement of observer agreement for categorical data. Biometrics 1977; 33:159-174.

53. Lugassy AA, Moffa JP. Laboratory model for the quantification of clinical occlusal wear. IADR program and abstracts 1985: Abstract no. 63.

54. Goldberg AJ, Rydinge E, Santucci EA, Racz WB. Clinical evaluation methods for posterior composite restorations. J Dent Res 1984;63:1387-91.

55. Mjör IA, Haugen E. Clinical evaluation of amalgam restorations. Scand J Dent Res 1976;84:333-7.

56. Elderton RJ. Assessment of the quality of restorations. J Oral Rehabil 1977;4:217-26.

57. Ryge G, Tonn EM, Eick JD, Jendresen MD. Evaluation of clinical behavior of six dental amalgams. IADR program and abstracts 1983:Abstract no. 62.

58. Bryant RW, Mahler DB, Engle JH. A comparison of methods for evaluating the marginal fracture of amalgam restorations. Dent Mater 1985;1:235-7.

59. Mahler DB, Engle JH, Bryant RW. Standardized evaluations of the clinical marginal fracture of amalgam J Dent Res 1986;65:1108-11. 
